\title{
Combined fluorescence and electrochemical investigation on the binding interaction between organic acid and human serum albumin
}

\author{
CHEN Yan-Min, GUO Liang-Hong* \\ State Key Laboratory of Environmental Chemistry and Ecotoxicology, Research Center for Eco-environmental Sciences, \\ Chinese Academy of Sciences, Beijing 100085, China. E-mail: 5237004@ rcees.ac.cn
}

Received 21 April 2008; revised 19 May 2008; accepted 23 May 2008

\begin{abstract}
Human serum albumin (HSA) is a plasma protein responsible for the binding and transport of fatty acids and a variety of exogenous chemicals such as drugs and environmental pollutants. Such binding plays a crucial role in determining the ADME (absorption, distribution, metabolism, and excretion) and bioavailability of the pollutants. The binding interaction between HSA and acetic acid (C2), octanoic acid (C8) and dodecanoic acid (C12) has been investigated by the combination of site-specific fluorescent probe, tryptophan intrinsic fluorescence and tyrosine electrochemistry. For the study of the fatty acid interaction with the two drug-binding sites on HSA, two fluorescent probes, dansylamide and dansyl- $L$-proline were employed in the displacement measurements. Intrinsic fluorescence of tryptophan in HSA was monitored upon addition of the fatty acids into HSA. Electrocatalyzed response of the tyrosine residues in HSA by a redox mediator was used to investigate the binding interaction. Qualitatively, observations from these three approaches were very similar. HSA did not show any change in the fluorescence and electrochemical experiments after mixing with $\mathrm{C} 2$, suggesting there is no significant interaction with the short-chain fatty acid. For C8, the measured signal dropped in a single-exponential mode, indicating an independent and non-cooperative binding. The calculated association constant and binding ratio were $3.1 \times 10^{6} \mathrm{~L} / \mathrm{mol}$ and $1 \mathrm{with}$ drug binding Site I, $1.1 \times 10^{7} \mathrm{~L} / \mathrm{mol}$ and 1 with Site II, and $7.0 \times 10^{4} \mathrm{~L} / \mathrm{mol}$ and 4 with the tryptophan site, respectively. The measurements with C12 displayed multiple phases of fluorescence change, suggesting cooperativity and allosteric effect of the C12 binding. These results correlate well with those obtained by the established methods, and validate the new approach as a viable tool to study the interactions of environmental pollutants with biological molecules.
\end{abstract}

Key words: absorption, distribution, metabolism, and excretion (ADME); organic acid; human serum albumin; fluorescence; electrochemistry

DOI: $10.1016 / \mathrm{S} 1001-0742(08) 62279-2$

\section{Introduction}

Human serum albumin (HSA) is the most abundant protein in blood plasma, typically is present at concentration around $0.6 \mathrm{mmol} / \mathrm{L}$ (Peter, 1995). The protein is a heart-shaped molecule composed of a single-chain of 585 amino acid residues with a molecular weight of $66400 \mathrm{Da}$. As first reported by Carter's group (Carter and Ho, 1994; He and Carter, 1992), HSA is made of three homologous domains (I, II and III), each with two subdomains (A and B). The main structural motif is $67 \%$ $\alpha$-helical, with 10 helices in each domain and four or six in each subdomain. The protein is responsible for the binding and transport of fatty acids, and also binds to a large variety of exogenous substances such as drugs and environmental chemicals. The interaction of these small molecules with HSA is important in determining their distribution, transport, metabolism and elimination in human body. Therefore, the study of such interaction is of great

\footnotetext{
* Corresponding author. E-mail: LHGuo@rcees.ac.cn
}

interest in the field of pharmakinetics, pharmatoxicology, and environmental toxicology.

The interaction of fatty acids with serum albumins has been studied extensively for over 40 years by a variety of techniques. Many researchers have contributed extensively to our knowledge of protein/fatty acid interaction (Hamilton, 2004; Choi et al., 2002; Curry et al., 1999; Simard et al., 2006). At present, the main method is the high-resolution X-ray diffraction crystallographic and nuclear magnetic resonance (NMR) method, which reveals the complete tertiary structure of the protein and molecular details of long-chain fatty acid and protein interactions. High-resolution X-ray crystal structure of HSA co-crystallized with fatty acids has been reported by several research groups (Curry et al., 1998; Bhattacharya et al., 2000; Petitpas et al., 2001). The study has mapped at least seven fatty acid binding sites for long-chain fatty acids (Bhattacharya et al., 2000; Petitpas et al., 2001). It has also found that the carboxyl head group of each fatty acid forms a salt bridge or a hydrogen bond with basic and polar amino acid side chains, and the tail fits into 
a hydrophobic cavity between helices (Hamilton, 2004). NMR studies by Hamilton's group reveal that among the seven fatty acid binding sites, sites 2, 4 and 5 are high affinity sites, while sites 1, 3, 6 and 7 exhibit low affinity for fatty acids (Simard et al., 2006). The unique ligand binding properties of HSA also include binding of a large number of exogenous compounds such as drugs. High-affinity drug binding usually takes place at drug site I or drug site II, which were first discovered by Sudlow et al. $(1975,1976)$. Drug site I (located in subdomain IIA) overlaps with fatty acid site 7 and drug site II (located in subdomain IIIA) overlaps with fatty acid sites 3 and 4 (Ghuman et al., 2005).

In addition to X-ray crystallography and NMR, a number of other techniques have been developed and employed in the study of drug-HSA interactions, such as ultrafiltration, equilibrium dialysis, calorimetry, circular dichroism, and fluorescence (Lee and McMenamy, 1980; Ashbrook et al., 1972, 1975; Kragh-Hansen, 1991; Tillement et al., 1974; Kragh-Hansen et al., 2001; Mahesha et al., 2006). Although the structural information is sometimes ambiguous, these techniques can provide complementary data on the dynamics of the interactions such as the binding constant with easy experimental protocols and relatively low-cost instruments. In particular, fluorescence technique provides a very high signal-to-noise ratio (Royer, 2006), and is exquisitely sensitive to the immediate environment of the fluorescent-emitting groups. Not surprisingly, fluorescence has become a widely used experimental technique in the investigation of HSA interaction with small molecules (Kragh-Hansen et al., 2001; Gelamo and Tabak, 2000; Gelamo et al., 2002; Vronique et al., 2005). In many these studies, the intrinsic fluorescence of the single tryptophan residue in HSA is used to monitor the protein conformation change upon binding.

We aim to employ a set of fluorescence and other easyto-use techniques to study the binding of environmental chemicals to proteins. The combination of several approaches can provide more definitive information on the binding interaction than any single one. In this report, octanoic acid (C8) is used as a model compound, which is known to bind with HSA. Two other fatty acids, acetic acid (C2) and dodecanoic acid (C12), are also evaluated and compared with $\mathrm{C} 8$ to examine the effect of chain length. Three different methods, namely site-specific fluorescent probe, tryptophan intrinsic fluorescence, and tyrosine electrochemistry, were employed in the investigation on the binding interaction between HSA and the fatty acids. The results obtained by the three approaches are qualitatively very similar, and also consistent with previous results by other methods. This validates our approach as a rapid and easy tool for the investigation of protein/pollutant interactions.

\section{Experimental}

Human serum albumin (fraction V, essentially fatty acid free, $\geqslant 96 \%$ purity) was purchased from SigmaAldrich Co. (USA). The protein was dissolved in a phosphate buffer made of $100 \mathrm{mmol} / \mathrm{L} \mathrm{NaH} \mathrm{PO}_{4} / 100$ $\mathrm{mmol} / \mathrm{L} \mathrm{Na} \mathrm{HPO}_{4}, \mathrm{pH} \mathrm{7.4}$, at a concentration of 100 $\mu \mathrm{mol} / \mathrm{L}$. The concentration of HSA was determined by UVVisible spectrophotomerty with an extinction coefficient $\left(\mathrm{E}_{1 \mathrm{~cm}}{ }^{1 \%}\right)$ of 5.3 at $280 \mathrm{~nm}$. The molecular weight of 66400 was used to calculate the molar concentration (Zia and Price, 1976). Acetic acid and dodecanoic acid were purchased from Beijing Chemicals Co. (China), and octanoic acid from Tianjin Chemicals Co. (China). Stock solutions of the fatty acids were prepared at the concentration of $200 \mathrm{mmol} / \mathrm{L}$ in ethanol. Dansylamide (DNSA) and dansyl$L$-proline (DP) were also purchased from Sigma-Aldrich Co. (USA). The stock solutions of DNSA and DP were prepared in acetonitrile.

Fluorescence emission spectra were measured on a Perkin-Elmer LS55 luminescence spectrometer. Tryptophan fluorescence of HSA was measured by exciting the protein solution at $295 \mathrm{~nm}$. Excitation wavelength of 295 nm was used to avoid the contribution from tyrosine residues. The emission spectra were recorded from 300 to $500 \mathrm{~nm}$. The excitation and emission slit width were both set to $15 \mathrm{~nm}$. Increasing concentrations of fatty acids ranging from 0 to $1 \mathrm{mmol} / \mathrm{L}$ were added to $\mathrm{HSA}$ from a $200 \mathrm{mmol} / \mathrm{L}$ stock solution in ethanol, and the fluorescence emission spectrum was recorded at each fatty acid concentration. Titration of a fatty acid against HSA with the binding site probes DNSA and DP were performed by varying the fatty acid concentration from 0 to $1 \mathrm{mmol} / \mathrm{L}$ while keeping both the protein and probe concentration constant at $0.1 \mathrm{mmol} / \mathrm{L}$. An excitation wavelength of 350 $\mathrm{nm}$ was used in the case of DNSA, and $375 \mathrm{~nm}$ for DP. The emission spectra were recorded from 400 to $600 \mathrm{~nm}$. Both the excitation and emission slit width were set at $15 \mathrm{~nm}$.

Indium tin oxide (ITO) conductive glass was obtained from WeiGuang Corp. (Shenzhen, China) and cut into size $2.5 \mathrm{~cm} \times 0.5 \mathrm{~cm}$ electrodes. The electrodes were cleaned in an ultrasonic cleaner sequentially with each of the following solutions: household detergent in water (15 min), deionized water (2 min, twice), acetone (5 $\mathrm{min}$ ), 2-propanol (5 min), and deionized water (10 $\mathrm{min}$, twice). Cyclic voltammetry was performed on a $\mathrm{CHI} 830 \mathrm{~B}$ electrochemistry analyzer (CH Instruments, Austin, USA) with a $\mathrm{Pt}$ counter electrode and an $\mathrm{Ag} / \mathrm{AgCl}$ reference electrode (also from CH Instruments, Austin, TX, USA). The position of the ITO electrode in the electrochemical cell was secured with a Teflon holder so that its area in contact with the electrolyte was fixed at $0.5 \mathrm{~cm}^{2}$.

\section{Results and discussion}

\subsection{Site-specific fluorescent probe}

As mentioned above in the Introduction Section, it has been generally accepted that, binding usually takes place at the two high-affinity sites, the so-called Sudlow's drug site I and site II although there are a number of ligand binding sites on HSA (Sudlow et al., 1975, 1976). Previous studies provide evidence that the binding affinity offered by the drug site I is mainly through hydrophobic interactions, whereas the site II involves a combination of hydrophobic, 
hydrogen bonding and electrostatic interactions (LhiaubetVallet et al., 2004; Jimenez et al., 2005). Moreover, the fatty acid binding site 4 is a high affinity binding site, while sites 3 and 7 exhibit low affinity for fatty acids (Simard et al., 2006). It has been shown that DNSA and DP are two fluorescence probes which are specific respectively for the two distinct drug binding sites on HSA (Pandey et al., 1997; Jisha et al., 2006). Therefore, the displacement of one of the probes from HSA by fatty acids would result in a reduction in fluorescence intensity, and could provide a measure of the binding specificity and relative strength of the fatty acids at a particular site.

As shown in Fig. S1 in Supporting Information, at 350 nm excitation wavelength free DNSA and HSA did not emit any fluorescence signal. But titration of DNSA into $0.1 \mathrm{mmol} / \mathrm{L} \mathrm{HSA}$ lead to a gradual increase in fluorescence with an emission peak at $478 \mathrm{~nm}$. The emission reached its highest value when DNSA concentration exceeded 0.1 $\mathrm{mmol} / \mathrm{L}$, and became steady afterwards. From the previous study of Scatchard and Ann (1949), the binding constant and binding ratio of DNSA to HSA are calculated to be $9.3 \times 10^{5} \mathrm{~L} / \mathrm{mol}$ and 0.68 , respectively. Similarly, at $375 \mathrm{~nm}$ excitation wavelength free DP and HSA have no fluorescence signal, while the DP/HSA complex has intensive fluorescence signal at $473 \mathrm{~nm}$ emission wavelength. From the titration curve illustrated in Fig. S2, the binding constant and binding ratio of DP to HSA are calculated to be $3.2 \times 10^{6} \mathrm{~L} / \mathrm{mol}$ and 0.47 , respectively. Previous study provides the binding constant and binding ratio of $1.7 \times 10^{5} \mathrm{~L} / \mathrm{mol}$ and 0.5 for DNSA to HSA, and $1.4 \times 10^{5}$ L/mol and 0.98 for DP to HSA, respectively (Sudlow et al., 1975). The discrepancy may arise from the different techniques employed in the measurements. In Sudlow's work, the binding constants and binding ratios of DNSA and DP were determined by competitive titration with drugs of known binding sites. Based on the above results, a molar ratio of 1:1 fluorescence probe/HSA complex was pre-formed in the displacement experiments to ensure all the HSA molecules were bound with the probe. A fatty acid of interest was then titrated into the complex. The result is displayed by a plot of $A / A_{0}$ as a function of the fatty acid concentration, where $A_{0}$ is the fluorescence of the probe/HSA complex before addition of the fatty acid, and $A$ is the fluorescence after addition of the fatty acid at a certain concentration.

Titration of C8 against HSA with the drug binding site I probe DNSA showed substantial reduction of the probe's fluorescence (Fig. 1a). At the low concentration of C8, the fluorescence intensity decreased and the peak wavelength red-shifted with increasing fatty acid concentrations. After titration of $0.1 \mathrm{mmol} / \mathrm{L} \mathrm{C8}$ (molar ratio of 1:1, C8:HSA), the fluorescence reduced to approximately $50 \%$ of its initial value, and the wavelength of the emission peak shifted from 478 to $486 \mathrm{~nm}$. Further addition of the fatty acid did not cause any change in either the intensity or the wavelength. The titration curve can be interpreted by invoking a competitive binding model in which C8 binds to the same site as the probe DNSA. Since free DNSA does not produce fluorescence, displacement of the probe by $\mathrm{C} 8$ from HSA reduces its fluorescence. The fact that the fluorescence did not reduce to zero even at high $\mathrm{C} 8$ concentrations (molar ratio of 10:1, C8:HSA) suggests there are other binding sites of the probe where the fatty acid can not compete. Similar results were obtained in the titration of $\mathrm{C} 8$ against HSA with the drug binding site II probe DP (Fig. 1b). The final fluorescence intensity decreased to $40 \%$ of the initial value, and peak wavelength $\lambda_{\max }$ shifted from 473 to $491 \mathrm{~nm}$. The binding affinity of the C8 fatty acid with HSA at the two drug binding sites is assessed by the $\mathrm{IC}_{50}$ approach (Cheng and Prusoff, 1973), which expresses the concentration of the competitor producing $50 \%$ signal inhibition. According to the displacement curve, and assuming complete displacement of the probe by $\mathrm{C} 8$ at the binding site under investigation condition, binding constants to drug sites I and II are estimated as $3.1 \times 10^{6}$ and $1.1 \times 10^{7} \mathrm{~L} / \mathrm{mol}$, respectively. The binding constants of C8 to HSA have been measured previously by equilibrium dialysis (Ashbrook et al., 1972; Kragh-Hansen, 1991), centrifugal ultrafiltration (Lee and McMenamy, 1980), and NMR (Kenyon and Hamilton,
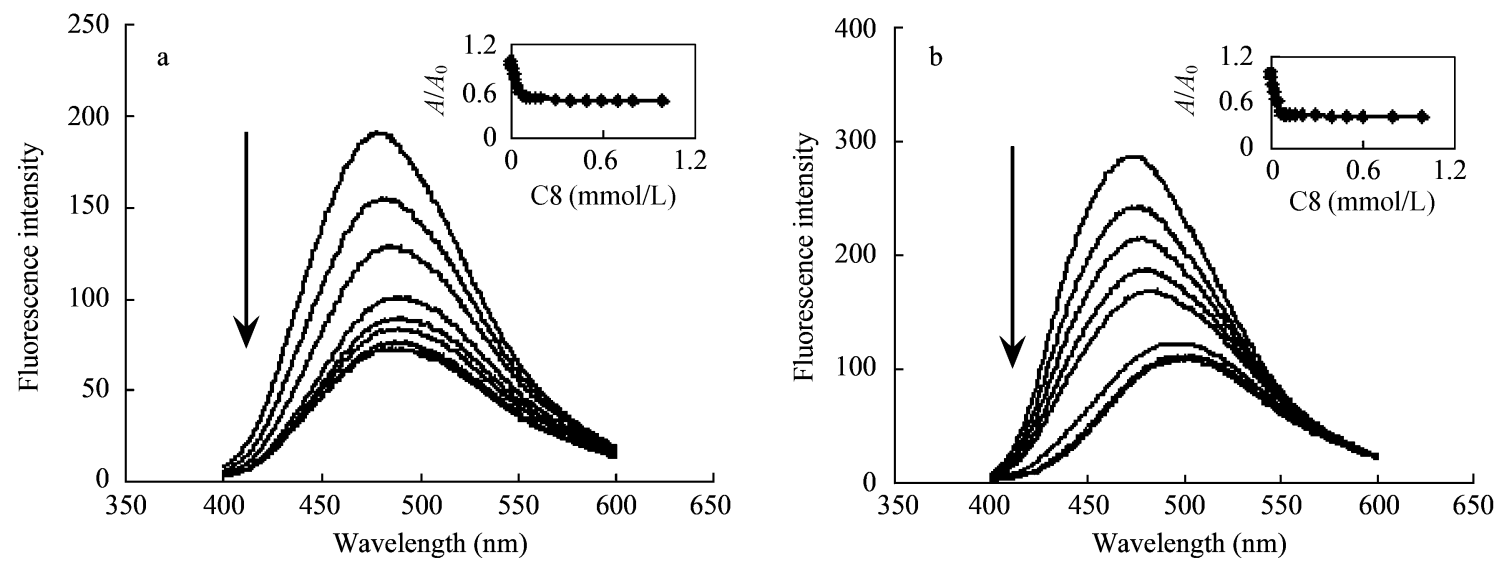

Fig. 1 Fluorescence displacement titration of C8 into (a) $0.1 \mathrm{mmol} / \mathrm{L} \mathrm{HSA}$ and $0.1 \mathrm{mmol} / \mathrm{L} \mathrm{DNSA}$, and (b) $0.1 \mathrm{mmol} / \mathrm{L} \mathrm{HSA}$ and $0.1 \mathrm{mmol} / \mathrm{L}$ DP. Conditions: (a) C8 concentration $0,0.02,0.04,0.08,0.16,0.3,0.6,0.8 \mathrm{mmol} / \mathrm{L}$, excitation wavelength $350 \mathrm{~nm}$, (b) C8 concentration $0,0.01,0.02,0.03$, $0.04,0.06,0.1,0.16 \mathrm{mmol} / \mathrm{L}$, excitation wavelength $375 \mathrm{~nm}$. Buffer: $100 \mathrm{mmol} / \mathrm{L}$ phosphate, $\mathrm{pH}$ 7.4. The arrow indicates increasing concentration of $\mathrm{C} 8$. Inset is a plot of the normalized fluorescence intensity as a function of $\mathrm{C} 8$ concentration. 
1994). The reported value for the highest binding site was $1.61 \times 10^{6}, 2.5 \times 10^{5}, 3.4 \times 10^{4}, 2.15 \times 10^{4} \mathrm{~L} / \mathrm{mol}$, respectively. The large discrepancy may reflect differences in experimental conditions and techniques used.

Titration of C12 against HSA with probe DNSA or DP exhibited multiple phases of fluorescence change, which is distinctively different from $\mathrm{C} 8$. At very low concentrations of $\mathrm{C} 12(0-0.04 \mathrm{mmol} / \mathrm{L})$, addition of the fatty acid lead to a reduction in DNSA fluorescence to $80 \%$ of the initial value (Fig. 2A). From 0.04 to $0.16 \mathrm{mmol} / \mathrm{L}$, the fluorescence intensity increased to $180 \%$ of the initial value. At higher concentrations of $\mathrm{C} 12$, a strong quenching of fluorescence took place. However, the peak position did not shift appreciably in the $\mathrm{C} 12$ concentrations range examined. The data suggest that $\mathrm{C} 12$ has several binding sites on HSA which modulate DNSA's fluorescence by different mechanisms. The rise in fluorescence from 0.04 to $0.16 \mathrm{mmol} / \mathrm{L} \mathrm{C12}$ is most likely due to the binding of the fatty acid at a site away from DNSA, resulting in conformational change of the protein that enhances the fluorescence of DNSA by allosteric effect. Reduction in fluorescence at low (0-0.04 mmol/L) and high $(0.15-$ $1 \mathrm{mmol} / \mathrm{L}$ ) $\mathrm{C} 12$ concentrations may be induced by the displacement of DNSA by the fatty acid, the quenching effect after conformational change, or a combination of both. Similarly, titration of C12 against HSA with probe DP also displayed three phases of fluorescence change (Fig. 2B).

Titration of C2 against HSA with probe DNSA or DP did not show any change in fluorescence, indicating the short-chain fatty acid does not interact strongly with the protein.

\subsection{Tryptophan intrinsic fluorescence}

Fluorescence spectroscopy has been used to monitor the changes on the tertiary structure of serum albumins induced by the interaction with drugs, fatty acids and other exogenous chemicals. HSA has a single fluorescent tryptophan residue $\left(\operatorname{Trp}^{214}\right)$ located in the hydrophobic cavity of the IIA subdomain in fatty acid binding site 7 , corresponding to the so-called drug binding site I region. The intrinsic fluorescence of the unique tryptophan reflects the environment around the amino acid residue. The binding interaction between HSA and the three fatty acids $(\mathrm{C} 2$, $\mathrm{C} 8$, and C12) was investigated by monitoring the $\operatorname{Trp}^{214}$ fluorescence in the fatty acid titration experiment.

As shown in Fig. 3, with C8 concentration increasing from 0 to $0.4 \mathrm{mmol} / \mathrm{L}$, tryptophan fluorescence was reduced progressively, and the emission peak wavelength blue-shifted from 342 to $333 \mathrm{~nm}$. Above $0.4 \mathrm{mmol} / \mathrm{L}$, both the emission intensity and the peak position became steady. The decrease in tryptophan fluorescence can be rationalized by the conformational change of the protein after $\mathrm{C} 8$ binding and subsequent quenching by other amino acid residues in the vicinity. Binding of the hydrophobic tail of the fatty acid into the cavity of HSA also increases the hydrophobicity in the surrounding of $\operatorname{Trp}^{214}$, leading to a shift of the emission peak to a shorter wavelength. From

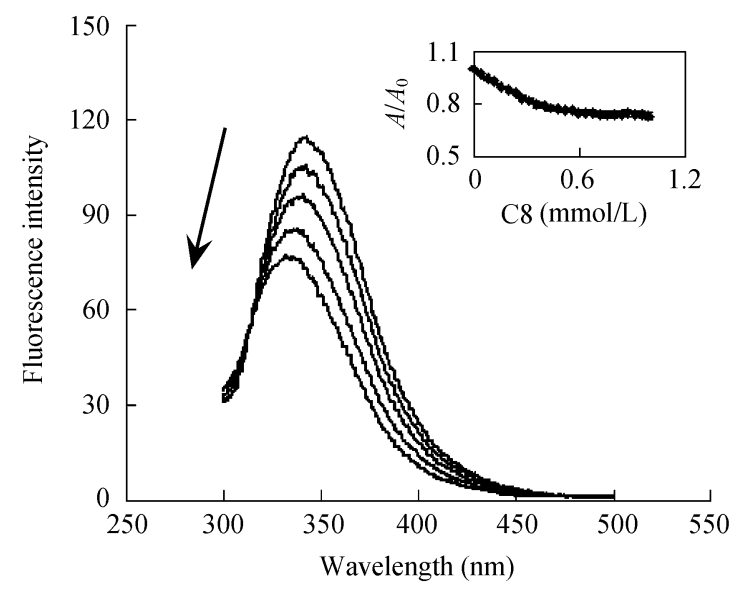

Fig. 3 Tryptophan fluorescence emission spectra of $0.1 \mathrm{mmol} / \mathrm{L} \mathrm{HSA}$

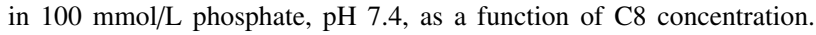
The arrow indicates increasing $\mathrm{C} 8$ concentration $(0,0.1,0.2,0.4,0.8$ $\mathrm{mmol} / \mathrm{L})$; excitation wavelength: $295 \mathrm{~nm}$. Inset is a plot of the normalized fluorescence intensity as a function of $\mathrm{C} 8$ concentration.
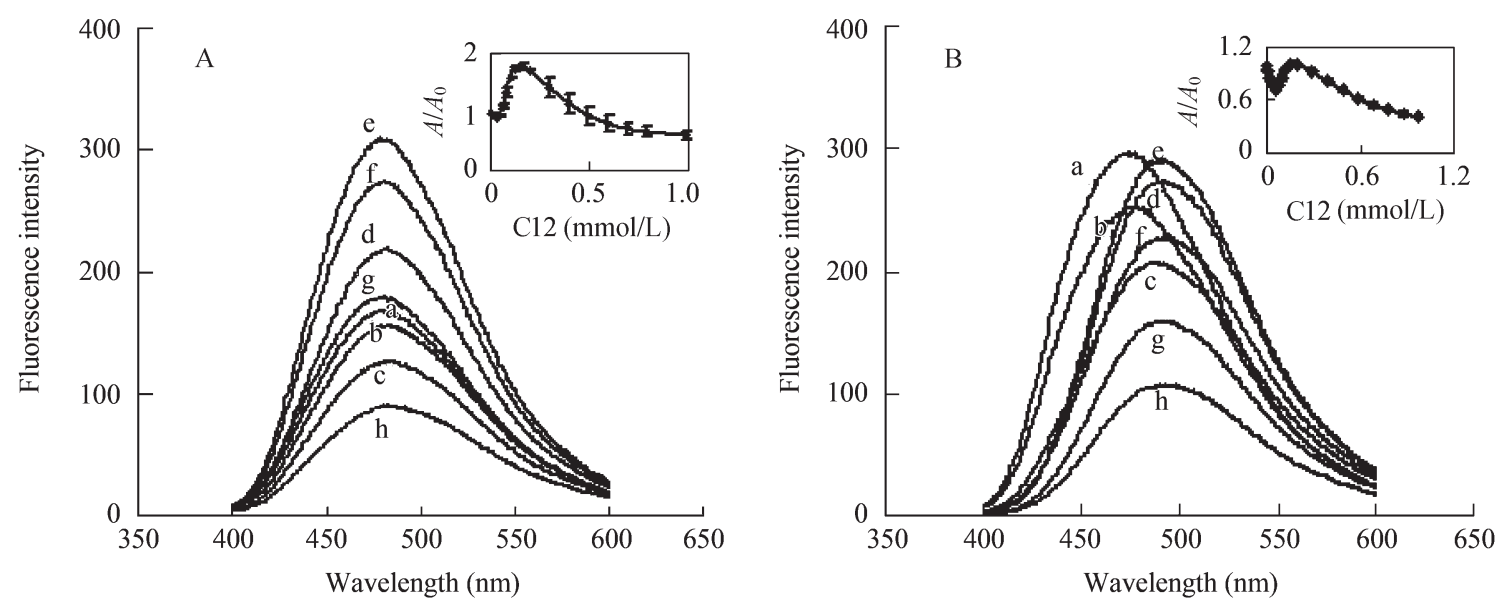

Fig. 2 Fluorescence displacement titration of C12 into (A) $0.1 \mathrm{mmol} / \mathrm{L} \mathrm{HSA}$ and $0.1 \mathrm{mmol} / \mathrm{L} \mathrm{DNSA}$, and (B) $0.1 \mathrm{mmol} / \mathrm{L} \mathrm{HSA}$ and $0.1 \mathrm{mmol} / \mathrm{L} \mathrm{DP}$. Conditions: (A) C12 concentration in alphabetic order $0,0.01,0.04,0.1,0.16,0.3,0.5,0.7 \mathrm{mmol} / \mathrm{L}$, excitation wavelength $350 \mathrm{~nm}$, (B) C12 concentration in alphabetic order 0, 0.02, 0.06, 0.11, 0.15, 0.4, 0.7, $0.9 \mathrm{mmol} / \mathrm{L}$, excitation wavelength $375 \mathrm{~nm}$. Buffer: $100 \mathrm{mmol} / \mathrm{L}$ phosphate, pH 7.4. Inset is a plot of the normalized fluorescence intensity as a function of $\mathrm{C} 12$ concentration. 
the plot of Scatchard and Ann (1949), an association constant of $7 \times 10^{4} \mathrm{~L} / \mathrm{mol}$ was obtained for the binding of $\mathrm{C} 8$ to HSA, with a binding ratio of 4 . The calculated binding constant is substantially smaller than those obtained using the two fluorescent probes, suggesting that the $\mathrm{C} 8$ binding site associated with $\operatorname{Trp}^{214}$ is different from drug sites I and II.

Compared with $\mathrm{C} 8$, addition of $\mathrm{C} 12$ into HSA produced only a slight decrease in tryptophan fluorescence, but with a significant blue-shift of $\lambda_{\max }$ from 342 to $328 \mathrm{~nm}$ at the $\mathrm{C} 12$ concentrations lower than $0.6 \mathrm{mmol} / \mathrm{L}$. At the $\mathrm{C} 12$ concentrations higher than $0.6 \mathrm{mmol} / \mathrm{L}$, there is no change in both of intensity and emission wavelength (Fig. 4). Comparing with $\mathrm{C} 8$, binding of $\mathrm{C} 12$ to HSA increases the hydrophobicity surrounding $\operatorname{Tr}^{214}$, which leads to a blueshift of the emission wavelength. But the lack of intensity change after $\mathrm{C} 12$ binding suggests the fatty acid interacts with the protein at some sites different from $\mathrm{C} 8$. This is consistent with the observation described above using the two extrinsic fluorescent probes. In a previous report, the interaction of dodecanoic acid with HSA was studied by tryptophan fluorescence (Kragh-Hansen et al., 2001). It was observed that at low dodecanoic acid concentrations, there was only a slight increase in fluorescence intensity and no change in peak position. At high concentrations, the fluorescence intensity increased further, accompanied by a blue-shift of $\lambda_{\max }$. Our results are somewhat different from the previous study, possibly due to the differences in buffer, $\mathrm{pH}$, fluorescence excitation wavelength, and source of HSA.

Consistent with the fluorescence probe experiments, titration of $\mathrm{C} 2$ into HSA did not induce any change to tryptophan fluorescence, suggesting there is no strong interaction between the two.

\subsection{Tyrosine electrochemistry}

HSA is a large single polypeptide protein (MW is $65 \mathrm{kDa}$ ) with 18 tyrosines and 1 tryptophan along with some cysteines, methionines and histidines that can po-

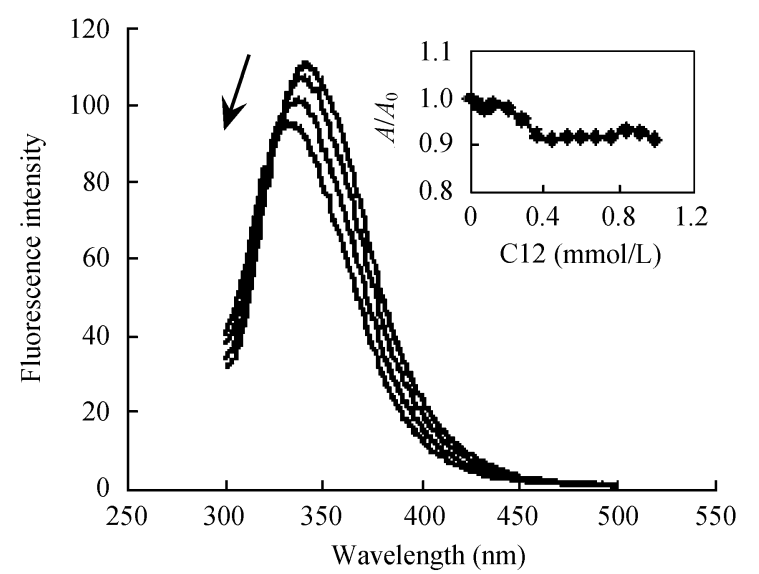

Fig. 4 Tryptophan fluorescence emission spectra of $0.1 \mathrm{mmol} / \mathrm{L}$ HSA

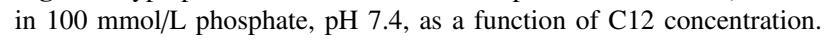
The arrow indicates increasing $\mathrm{C} 12$ concentration $(0,0.2,0.36,0.6$ $\mathrm{mmol} / \mathrm{L}$ ); excitation wavelength: $295 \mathrm{~nm}$. Inset is a plot of the normalized fluorescence intensity as a function of $\mathrm{C} 12$ concentration. tentially be oxidized. But on most electrode surfaces the electrochemical response of these amino acids is slow and irreversible (Brown et al., 1977). We have previously developed an electro-catalytic system to selectively and reversibly catalyze the oxidation of tyrosines in bovine serum albumin (BSA) on a indium tin oxide electrode using an osmium tris(bipyridine) (Os-bipy) complex as an electron mediator (Guo and Qu, 2006). Because most of the non-polar tyrosine residues are located in the interior of the water-soluble protein, access of the electron mediator to the tyrosine sites is critically dependent on the conformation of the protein. Therefore, its electro-catalytic current was used in that previous study (Guo and Qu, 2006)as a signal to monitor the unfolding of BSA induced by a chemical denaturant, urea.

It was anticipated that binding of fatty acids to HSA could also modulate the electrochemical response of tyrosine by blocking the diffusion of Os-bipy to some of the amino acid sites. Similar to BSA, although HSA itself produced a low and irreversible oxidation wave on an ITO electrode, the current was catalyzed by almost 5-fold in the presence of $5 \mu \mathrm{mol} / \mathrm{L}$ Os-bipy. The increased electrochemical signal permits the binding study to be carried out at low protein and fatty acid concentrations, which is necessary due to the poor solubility of the fatty acids in water. As in the fluorescence experiments, fatty acids were titrated into $0.1 \mathrm{mmol} / \mathrm{L} \mathrm{HSA}$, and the catalyzed electrochemical current was measured. As shown in Fig. 5, the current decreased with the increase of $\mathrm{C} 8$ concentrations. At the highest fatty acid concentration examined ( $1 \mathrm{mmol} / \mathrm{L})$, the current was reduced to $58 \%$ of its original value. However, when the titration experiment was repeated in the presence of $8 \mathrm{~mol} / \mathrm{L}$ urea, the current stayed unchanged regardless of the C8 concentration added into HSA solution (Fig. 6). Presumably, some of the tyrosine residues are located in the cavity inside HSA for binding with $\mathrm{C} 8$, through which the electron mediator diffuses and catalyzes the tyrosine oxidation. After C8 binding, the cavity is occupied by the hydrophobic tail of the fatty acid, and diffusion of

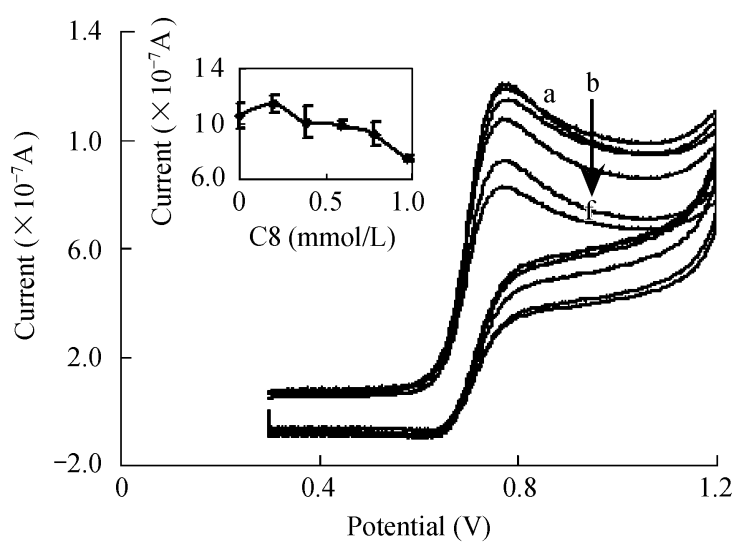

Fig. 5 Cyclic voltammograms of $100 \mu \mathrm{mol} / \mathrm{L}$ HSA in the presence of 5 $\mu \mathrm{mol} / \mathrm{L} \mathrm{Os}(\mathrm{bpy})_{3}{ }^{2+}$ with addition of (a) 0, (b) 0.2, (c) 0.4, (d) 0.6, (e) 0.8, and (f) $1.0 \mathrm{mmol} / \mathrm{L} \mathrm{C} 8$. Conditions: working electrode, indium tin oxide $\left(0.5 \mathrm{~cm}^{2}\right)$; reference electrode, $3 \mathrm{~mol} / \mathrm{L} \mathrm{Ag} / \mathrm{AgCl}$; supporting electrolyte, $100 \mathrm{mmol} / \mathrm{L}$ phosphate, $\mathrm{pH} 7.4$; scan rate, $30 \mathrm{mV} / \mathrm{s}$. Inset is a plot of the catalyzed oxidation current as a function of $\mathrm{C} 8$ concentration. 


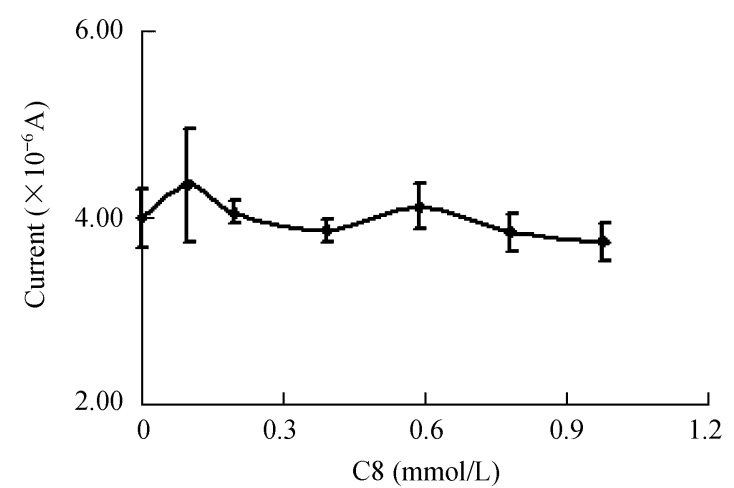

Fig. 6 The catalyzed oxidation current of $0.1 \mathrm{mmol} / \mathrm{L}$ urea-denatured HSA in the presence of $5 \mu \mathrm{mol} / \mathrm{L} \mathrm{Os(bpy)})^{2+}$ as a function of C8 concentration. Conditions: working electrode, indium tin oxide $(0.5$ $\mathrm{cm}^{2}$ ); reference electrode, $3 \mathrm{~mol} / \mathrm{L} \mathrm{Ag} / \mathrm{AgCl}$; supporting electrolyte, 100 $\mathrm{mmol} / \mathrm{L}$ phosphate $(\mathrm{pH} 7.4)$; scan rate, $30 \mathrm{mV} / \mathrm{s}$.

the mediator inside the protein is impeded. As a result, the electro-catalytic reaction slows down. In the presence of urea, the protein acquires a random-coil structure, and loses its specific binding sites for fatty acids. Therefore, addition of C8 into HSA does not initiate any interaction between the two, and thereby has no effect on tyrosine electrochemistry. Because the drop in current did not reach a plateau, binding constant could not be estimated.

Different from $\mathrm{C} 8$, addition of $\mathrm{C} 12$ into native HSA did not cause any change in the catalyzed oxidation current of the protein. Although currently there is no hypothesis to rationalize the observation, the result demonstrates once again that the binding modes of $\mathrm{C} 12$ are distinctively different from $\mathrm{C} 8$, as already revealed by the two fluorescence experiments described above. In addition, the data of $\mathrm{C} 2$ show that this chemical has no effect on the oxidation current of either native or urea-denatured HSA.

\section{Conclusions}

Our study shows that a combination of fluorescence and electrochemistry methods can be very useful in investigating the molecular interactions between organic acids and HSA. The results of fluorescence and electrochemistry indicate that acetic acid (C2) has no interaction with HSA, whereas octanoic acid (C8) and dodecanoic acid (C12) have distinct profiles in terms of binding site and strength to HSA. The differences in binding strength for the three fatty acids are largely consistent with previous reports which have shown that binding constants increase with fatty acid chain length. The chain-length dependence can be attributed to the increased interaction between the hydrocarbon tail of long-chain fatty acids and the hydrophobic cavity at the binding site of HSA (Hamilton, 2004). From the fluorescence measurements, the binding constant and binding ratio of $\mathrm{C} 8$ at three distinctive sites of the protein was determined as $1.1 \times 10^{7} \mathrm{~L} / \mathrm{mol}(n=1), 3.1 \times 10^{6} \mathrm{~L} / \mathrm{mol}$ $(n=1)$ and $7 \times 10^{4} \mathrm{~L} / \mathrm{mol}(n=4)$, respectively. The results from the fluorescence and electrochemistry methods correlate well with the established high-resolution X-ray diffraction crystallographic and NMR data. The combined approach can be employed to investigate the interaction between other environmental chemicals and proteins.

\section{Acknowledgments}

This work was supported by the National Basic Research Program of China (No. 2006CB403303), the Knowledge Innovation Program of the Chinese Academy of Sciences (No. KZCX2-YW-420-1), and the National Natural Science Foundation of China (No. 20890112)

\section{Supporting materials}

Details of the titration experiment of site-specific fluorescent probe DNSA and DP with HSA.

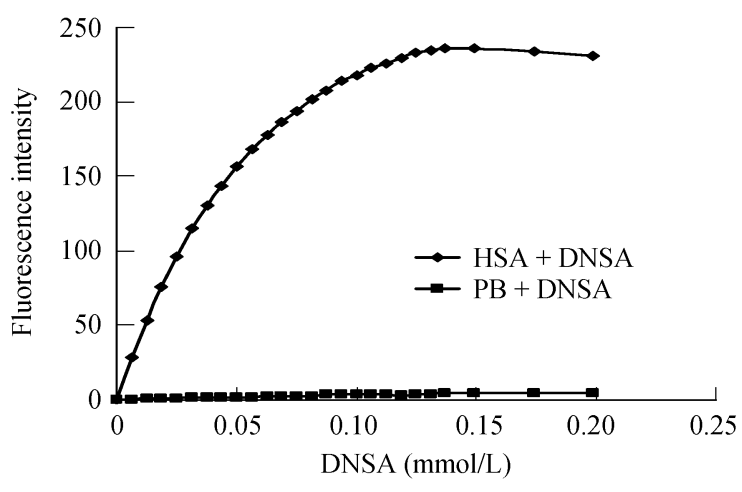

Fig. S1 Fluorescence titration of DNSA into $0.1 \mathrm{mmol} / \mathrm{L} \mathrm{HSA}$ in 100 $\mathrm{mmol} / \mathrm{L}$ phosphate, $\mathrm{pH}$ 7.4. Excitation wavelength: $350 \mathrm{~nm}$.

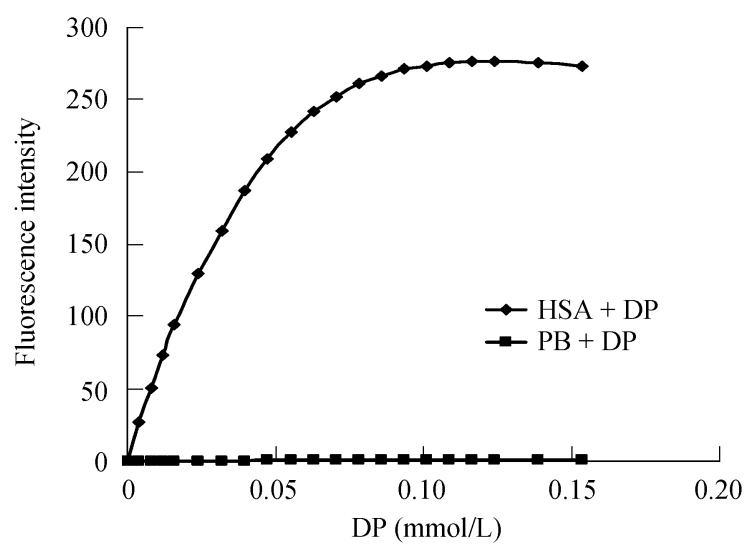

Fig. S2 Fluorescence titration of DP into $0.1 \mathrm{mmol} / \mathrm{L} \mathrm{HSA}$ in $100 \mathrm{mmol} / \mathrm{L}$ phosphate, $\mathrm{pH}$ 7.4. Excitation wavelength: $375 \mathrm{~nm}$.

\section{References}

Ashbrook J D, Spector A A, Fletcher J E, 1972. Medium-chain fatty acid binding to human plasma albumin. Journal of Biological Chemistry, 247(21): 7038-7042.

Ashbrook J D, Spector A A, Santos E C, Fletcher J E, 1975. Long chain fatty acid binding to human plasma albumin. Journal of Biological Chemistry, 250(6): 2333-2338.

Bhattacharya A A, Grune T, Curry S, 2000. Crystallographic analysis reveals common modes of binding of medium and long-chain fatty acids to human serum albumin. Journal of Molecular Biology, 303(5): 721-732. 
Brown J R, Rosenoer V M, Oratz M, Rothschild M A, 1977. In Albumin Structure, Function and Uses. Oxford, UK: Pergamon Press.

Carter D C, Ho J X, 1994. Structure of serum albumin. Advances in Protein Chemistry, 45: 152-203.

Cheng Y C, Prusoff W H, 1973. Relationship between the inhibition constant $\left(K_{i}\right)$ and the concentration of inhibitor which causes 50 per cent inhibition $\left(I_{50}\right)$ of an enzymatic reaction. Biochemical Pharmacology, 22(23): 3099-3108.

Choi J, Ho K J, Curry S, Qin D H, Bittman R, Hamilton J A, 2002. Interactions of very long-chain saturated fatty acids with serum albumin. Journal of Lipid Research, 43(7): 10001010 .

Curry S, Brick P, Franks N P, 1999. Fatty acid binding to human serum albumin: new insights from crystallographic studies. Biochimica et Biophysica Acta, 1441(2): 131-140.

Curry S, Mandelkow H, Brick P, 1998. Crystal structure of human serum albumin complexed with fatty acid reveals an asymmetric distribution of binding sites. Nature Structure Biology, 5(8): 827-835.

Gelamo E L, Silva C H T P, Imasato H, Tabak M, 2002. Interaction of bovine (BSA) and human (HSA) serum albumins with ionic surfactants: spectroscopy and modeling. Biochimica et Biophysica Acta, 1594(1): 84-99.

Gelamo E L, Tabak M, 2000. Spectroscopic studies on the interaction of bovine (BSA) and human (HSA) serum albumins with ionic surfactants. Spectrochimica Acta Part A, 56(11): 2255-2271.

Ghuman J, Zunszain P A, Petitpas I, Bhattacharya A A, Otaqiri M, Curry S, 2005. Structural basis of the drug-binding specificity of human serum albumin. Journal of Molecular Biology, 353(1): 38-52.

Guo L H, Qu N, 2006. Chemical-induced unfolding of cofactorfree protein monitored by electrochemistry. Analytical Chemistry, 78(17): 6275-6278.

Hamilton J A, 2004. Fatty acid interactions with proteins: what $\mathrm{X}$-ray crystal and NMR solution structures tell us. Progress in Lipid Research, 43(3): 177-199.

He X M, Carter D C, 1992. An abundant protein found in the circulatory system that aids in the transport of many ligands. Nature, 358(6383): 209-215.

Jimenez M C, Miranda M A, Vaya I, 2005. Triplet excited states as chiral reporters for the binding of drugs to transport proteins. Journal of the American Chemical Society, 127(29): 10134-10135.

Jisha V S, Arun K T, Hariharan M, Ramaiah D, 2006. Siteselective binding and dual mode recognition of serum albumin by a squaraine dye. Journal of the American Chemical Society, 128(18): 6024-6025.

Kenyon M A, Hamilton J A, 1994. C-13 NMR-studies of the binding of medium-chain fatty-acids to human serumalbumin. Journal of Lipid Research, 35(3): 458-467.

Kragh-Hansen U, 1991. Octanoate binding to the indole- and benzodiazepine-binding region of human serum albumin.
Biochemical Journal, 273(3): 641-644.

Kragh-Hansen U, Hellec F, de Foresta B, le Maire M, Møller J V, 2001. Detergents as probes of hydrophobic binding cavities in serum albumin and other water-soluble proteins. Biophysical Journal, 80(6): 2898-2911.

Lee I Y, McMenamy R H, 1980. Location of the medium chain fatty acid site on human serum albumin. Residues involved and relationship to the indole site. Journal of Biological Chemistry, 255(13): 6121-6127.

Lhiaubet-Vallet V, Sarabia Z, Bosca F, Miranda M A, 2004. Human serum albumin-mediated stereodifferentiation in the triplet state behavior of $(S)$ - and $(R)$-carprofen. Journal of the American Chemical Society, 126(31): 9538-9539.

Mahesha H G, Singh S A, Srinivasan N, Appurao A G, 2006. A spectroscopic study of the interaction of isoflavones with human serum albumin. FEBS Journal, 273(3): 451-467.

Pandey R K, Constantine S, Tsuchida T, Zheng G, Medforth C J, Aoudia M, Kozyrev A N, Rodgers M A J, Kato H, Smith K M, Dougherty T J, 1997. Synthesis, photophysical properties, in vivo photosensitizing efficacy and human serum albumin binding properties of some novel bacteriochlorins. Journal of Medicinal Chemistry, 40(17): 2770-2779.

Peter T J, 1995. All about Albumin: Biochemistry, Genetics, and Medical Applications. New York, USA: Academic Press.

Petitpas I, Grune T, Bhattacharya A A, Curry S, 2001. Crystal structures of human serum albumin complexed with monounsaturated and polyunsaturated fatty acids. Journal of Molecular Biology, 314(5): 955-960.

Royer C A, 2006. Probing protein folding and conformational transitions with fluorescence. Chemical Review, 106(5): 1769-1784.

Scatchard G, 1949. The attractions of proteins for small molecules and ions. Annals of the New York Academy of Sciences, 51: 660-663.

Simard J R, Zunszain P A, Hamilton J A, 2006. Location of high and low affinity fatty acid binding sites on human serum albumin revealed by NMR drug-competition analysis. Journal of Molecular Biology, 361(2): 336-351.

Sudlow G, Birkett D J, Wade D N, 1975. The characterization of two specific drug binding sites on human serum albumin. Molecular Pharmacology, 11(6): 824-832.

Sudlow G, Birkett D J, Wade D N, 1976. Further characterization of specific drug binding sites on human serum albumin. Molecular Pharmacology, 12(6): 1052-1061.

Tillement J R, Zini R, D'athis P, Vassent G, 1974. Binding of certain acidic drugs to human albumin: theoretical and practical estimation of fundamental parameters. European Journal of Clinical Pharmacology, 7: 307-313.

Véronique P, Virginie L, Virginie A, 2005. Detergent binding as a sensor of hydrophobicity and polar interactions in the binding cavities of proteins. Langmuir, 21(19): 8865-8875.

Zia H, Price J C, 1976. Binding study of tetracyclines to human serum albumin using difference spectrophotometry. Journal of Pharmaceutical Sciences, 65(2): 226-230. 